\title{
Ciprofloxacin-Induced Allergic Interstitial Nephritis
}

\begin{tabular}{|l|l|l|}
\hline R. & Rosemarie & Helmink $^{\mathrm{a}}$ \\
\hline H. & Hallgrimur & Benediktsson $^{\mathrm{b}}$ \\
\hline
\end{tabular}

${ }^{a}$ Red Deer Regional Hospital Centre, Red Deer, Alta., and ${ }^{\mathrm{b}}$ Foothills Hospital, Calgary, Alta., Canada

Dr. Rosemarie Helmink, Clinical Coordinator, Pharmacy, Red Deer Regional Hospital Centre, PO Bag 5030, Red Deer, Alta. (Canada) T4N 6R2

Dear Sir,

Ciprofloxacin has been implicated as a cause of both documented [1, 2] and suspected [3, 4] allergic interstitial nephritis. We report another case of nephrotoxicity likely induced by ciprofloxacin.

A 73-year-old man had an aortobifemoral graft 10 years ago. At the present admission, arterial blood collected distal to the graft grew Staphylococcus epidermidis sensitive to methicillin. Therapy with intravenous cloxa-cillin was initiated. A laparotomy was performed. The femoral arteries and an aortic false aneurysm were oversewed, a bilateral axilloprofundal bypass of GortexR was placed, and a portion of bowel was resected because of an impending aortoduodenal fistula. Intravenous cloxacil-lin was continued postoperatively and augmented with parenteral gentamicin and metronidazole. Four days after surgery, sputum was cultured and grew Pseudomonas aeruginosa resistant to gentamicin. Aminoglycoside therapy was switched to tobramycin, and the dosage was adjusted to maintain therapeutic concentrations (peak 8.0-10.0 $\mathrm{mg} / \mathrm{l}$, trough $<2.0 \mathrm{mg} / \mathrm{l}$ ). Eighteen days after surgery, arterial blood collected below the new graft grew S. epidermidis resistant to methicillin. Cloxacillin was replaced with vancomycin, its dosage being adjusted to maintain therapeutic concentrations (peak 30.0-40.0 mg/l, trough 5.0$10.0 \mathrm{mg} / \mathrm{l}$ ). The nephrotoxicpotential of tobramycin and vancomycin prompted a request for ciprofloxacin which had investigational status in Canada at the time. The other antibiotics were discontinued, and

ciprofloxacin $200 \mathrm{mg}$ i.v. every $12 \mathrm{~h}$ was initiated. On the 3rd day of therapy, an erythematous rash developed on the patient's trunk and limbs. Ciprofloxacin was continued 11 days, and the rash persisted. During the period of ciprofloxacin therapy, intermittent doses of fenoterol, morphine, and furosemide were administered. The day prior to ciprofloxacin therapy, serum creatinine was $128 \mu \mathrm{mol} / \mathrm{l}$ and the white blood cell count (WBC) was $22.9 \times 10 \mathrm{~V} 1$ with $1 \%$ eosinophils. On day 2 of ciprofloxacin therapy, serum creatinine increased to $178 \mu \mathrm{mol} / \mathrm{l}$, and the WBC was $15.8 \times 10 \mathrm{~V} 1$ with $6 \%$ eosinophils. On day 11 of ciprofloxacin, creatinine was 292 $\mu \mathrm{mol} / \mathrm{l}$, and the WBC was $33.2 \times 10 \mathrm{~V} 1$ with $15 \%$ eosinophils. There was never oliguria. On day 11 , the patient died.

Autopsy disclosed bronchopneumonia and emphysema. The kidneys showed interstitial inflammation with prominent eosinophils and focal tubular destruction. The histologic appearance was that of interstitial nephritis, consistent with drug allergy. 
The sequence of events indites ciprofloxacin as the agent responsible for the pathology.

Although tobramycin has caused renal toxicity, acute tubular necrosis is the typical lesion [5]. The nephrotoxic potential of vancomycin has been doubted [6], but this drug may enhance the inherent toxicity of aminoglycosides [7]. However, both agents had been discontinued several days prior to evidence of renal dysfunction.

Nephrotoxicity with quinolone therapy appears to be a rare phenomenon. A review of the literature revealed no documentation of this adverse effect in association with Ciprofloxacin-Induced Allergic Interstitial Nephritis

433

the clinical use of nalidixic acid or norfloxacin. Prolonged high dosages of ofloxacin, pefloxacin, or enox-acin in animals have caused, respectively, tubular atrophy, interstitial nephritis, and tubular dilatation with pigmentation [8].

References

Reppelmeyer DJ, Synhavsky A: Ciprofloxacin and allergic interstitial nephritis. Ann Intern Med 1988; 109:170.

Ying LS, Johnson CA: Ciproñoxacin induced interstitial nephritis. Clin Pharm 1989;8:518-521. Campoli-Richards DM, Monk JP, Price A, Benfïeld B, Todd PA, Ward A: Ciprofloxacin. Drugs 1988;35:373-447.

Arciere G, Griffith E, Gruenwaldt G, et al: Ciprofloxacin: An update on clinical experience. Am J Med 1987; 82 (suppl 4A): 381-386.

Sande MA, Mandell GL: Antimicrobial agents: The aminogly-cosides; in Goodman Gilman A, Goodman LS, Rail TW, Murad F(eds): The Pharmacological Basis of Therapeutics. New York, Macmillan, 1985, p 1159.

Appel GB, Neu HC: The nephrotoxicity of antimicrobial agents. N Engl J Med 296; 1977:722_ 728.

Rybak MJ, Boike SC: Monitoring vancomycin therapy. Drug Intell Clin Pharm 1986;20:150_ 153. 